Journal of Advanced Research in Applied

Sciences and Engineering Technology

Journal homepage: www.akademiabaru.com/araset.html ISSN: 2462-1943

\title{
A Brief Review of Particle Dispersion of Cavity Flow
}

\author{
Ahmad Sofianuddin A. Sahak ${ }^{1}$, Nor Azwadi Che Sidik ${ }^{1,}{ }^{*}$, Siti Nurul Akmal Yusof ${ }^{1}$ \\ Malaysia - Japan International Institute of Technology (MJIT), University Teknologi Malaysia, Jalan Sultan Yahya Petra,54100 Kuala Lumpur, \\ Malaysia
}

\begin{tabular}{|c|c|}
\hline ARTICLE INFO & ABSTRACT \\
\hline $\begin{array}{l}\text { Article history: } \\
\text { Received } 29 \text { June } 2020 \\
\text { Received in revised form } 11 \text { October } 2020 \\
\text { Accepted } 20 \text { October } 2020 \\
\text { Available online } 11 \text { November } 2020\end{array}$ & $\begin{array}{l}\text { Particles dispersion studies have attracted researchers' interest in understanding the } \\
\text { physics behind the phenomena. Fluid-particle transport related to cavity flow is } \\
\text { relevant to several natural and engineering applications such as cleaning of process } \\
\text { equipment. This paper intends to provide a brief review of researches on the impact } \\
\text { parameters, including geometrical parameters of the cavities, flow-related } \\
\text { parameters, heat transfer and fluid-particles interaction and identifies opportunities } \\
\text { for future research. }\end{array}$ \\
\hline
\end{tabular}

Keywords:

Particle dispersion, cavity flow, geometrical parameter

Copyright @ 2020 PENERBIT AKADEMIA BARU - All rights reserved

\section{Introduction}

\subsection{Fundamental Concepts of Cavity Flow}

In general, the phrase cavity flow refers to flow over the surface cavity or surface cut-outs. Cavity flows relevant to study the flow regime without implementing complicated geometric structure. Figure 1 shows a general cavity two-dimensional configuration for a rectangular cavity, where $H$ is the depth of the cavity and $L$ is the length of the cavity. The dimensions of a cavity are asserted by their aspect ratio $(A R)$. In the analysis of fluid flow, the important things to identify the types of flow related to the scope of studies. In fluid dynamics, fluid flow can be classified into several types which are I) laminar or turbulent, II) compressible or incompressible, III) uniformed or non-uniformed, IV) steady or unsteady, V) rotational or non-rotational and VI) two or three-dimensional flow. The dimensionless parameter to investigate the type of fluid flow is $R e$.

Primarily, there are two types of inlet flow in the channel, which is Poiseuille flow and Couette flow. Poiseuille flow is a flow guided by a pressure gradient along the channel length with a usually fixed cross-section Couette flow is a viscous fluid that moves tangentially in the space between two surfaces as shown in Figure 2.

\footnotetext{
* Corresponding author.

E-mail address: azwadi@utm.my

https://doi.org/10.37934/araset.20.1.2741
} 
Incoming flow

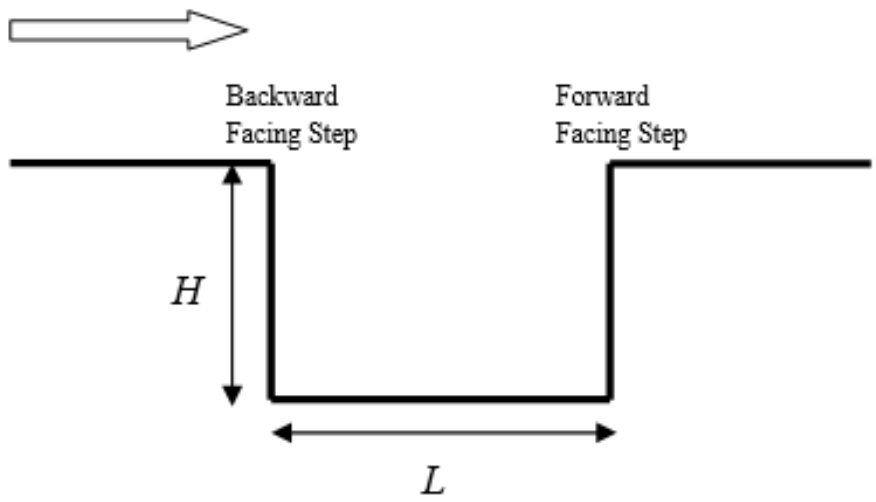

Fig. 1. General two-dimensional cavity flow configuration

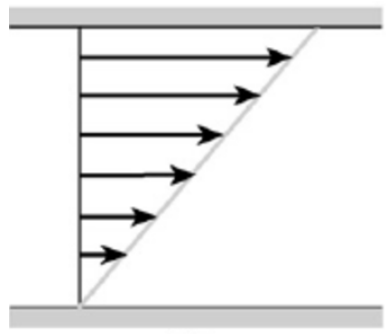

(a)



$\stackrel{x}{\longrightarrow}$

Fig. 2. The fluid flow of the plane shear (a) Couette flow with upper wall moving and (b) Poiseuille flow with both walls fixed where the arrows show the velocity magnitude of the flow [1]

There are no specific classifications of type of flow formed inside the cavity. However, there are classification made based on the application, and problem researchers try to solve. For example, in the study of airflow in building and street canyon, the flow regime in the urban boundary layer can be categorized into three types: isolated roughness flow, wake interference flow and skimming flow regime [2]. The development of the flow regime in the urban street canyon depends on the flow characteristics, the height of the building and the distance between two buildings [3].

On the other hand, Charwat et al., [4] introduced a classic definition of different flow regimes. In general, there are two types of cavity flow which are "open" cavity flow and "closed" cavity flow. Between these two types of flow, the transitional flow region also was put into the categories. These categories are based on a two-dimensional rectangular cavity where the spanwise width, $W$ is much larger than the length of the cavity, $L$ and end effect can be ignored.

Particles dispersion studies have attracted researchers' interest in understanding the physics behind the phenomena. Fluid-particle transport related to cavity flows is relevant to several natural and engineering applications such as cleaning of process equipment. However, there is no detailed discussion on the flow in the cavity channel with the dispersion of the particles, and the combination of the effect of different geometrics and fluid parameters with the presence of heat. The study presented here discusses the previous work of several researchers on the impact parameters, including geometrical parameters of the cavities, flow-related parameters, heat transfer and fluidparticles interaction. In addition, application of CIP method in this area of studies is considered new. This is therefore, form the foundation for the objectives and the significance of the current studies. 


\subsection{Cavity Geometrical Effects}

One of the parameters that affected flow structure inside the cavity is its geometry. As the aspect ratio, $A R$ of cavities changes, the flow regime inside the cavity also changes. There are several studies in which the results highlight the effect of geometrical parameters on the flow inside the cavity. Earlier, Pan and Acrivos [5] studied the dependence on the depth of cavities and the Stokes flow eddy structure in a closed, rectangular cavity. These natural occurrences can theoretically be clarified by the development of their value for stream function, $\psi(x, y)$ as explained by Shankar and Deshpande [6](2000). For mathematical details of this matter, Joseph et al., [7] worked can be used as a reference. Furthermore, the formation of the primary vortex from the corners eddies as the changes of cavity depth have been visualized in detail by Hellou and Coutanceau [8].

Cheng and Hung [9] studied the effect of the deep cavity to the vortex structure from $A R=1$ to 1/7. For deep cavity, the number of vortices is increased with both depth, $H$ and Reynolds number, $R e$. The main feature of the deep cavity is its fill with counter-rotating vortices. There is a critical value of the aspect ratio for a deep cavity which is depending on the $R e$ [10]. When exceeding the significant value, flow structure in a certain part of the cavity becomes symmetric due to loss of inertial forces from the primary vortex at the top of the cavity from reach to the bottom.

In the deep cavity, the evolution of the secondary or tertiary vortex can be seen as a function of the depth of the cavity $[9,11]$. As the $H$ of the cavity increases, the separation point between two secondary vortices in the bottom corner of the cavity decreasing and eventually attached become one large vortex. For $R e=0.01$, the attachment occurs when $H=1.63$. Mehta and Lavan [12] observed two vortices formed, where one formed at the bottom from the first one for $R e$ ranging from 1 to 100 at $A R=0.5$. The first top vortex is seemed to be appearing stronger from the other one. As the $A R$ decrease to 0.2 , four single large vortices were identified [5].

As $A R$ decreases, the vortices number in the cavity increases. However, the strength of vortices reduces rapidly [13]. Only primary and secondary vortices in the deep cavity promote mass transfer. The values of kinetic energy in the primary vortex is high, therefore has large influence on mass transfer. The impact of secondary vortex reduces quickly as $A R$ decrease. The other vortices in the deep cavity therefore have a negligible effect on the transfer of mass.

For a study on the flow over shallow cavity, Taneda [14] investigated the effect of the $A R$ on the laminar regime, and results have been numerically verified by Shen and Floryan [15]. They studied low $R e$ for various $A R$ numerically and experimentally. They found that corner vortices or Molfatt vortices existed regardless of the aspect ratio. Furthermore, the location of impingement and separation points correlated with $A R[16]$.

Zdanski et al., [17] studied for both laminar and turbulent flow over the shallow cavity. For laminar flow study, they considered cavity for $A R$ ranging from 9.6, 10 and 28 and $R e$ was kept constant at 662. The finding shows the cavity's $A R$ influencing to the number and shape of the recirculating zone. The separation of the vortex start occurred at $A R=10$ when the formation of two vortices and as $A R$ is increasing to 28 , two vortices formed at forward and backwards-facing step of the channel.

Moreover, Cheng and Hung [9] highlighted that a large vortex that fills near the cavity region is the main feature. Furthermore, the formation of small vortices can be seen at the bottom corner of the cavity. This feature, however, can be seen for very low $R e$ or creeping flow. The effect of different $R e$ will be discussed in the next section. Besides, for $R e \leq 100$, the streamline pattern and vortex structure are discovered to be almost identical. However, it can be seen that the primary vortex seems to appear more circular as the $L$ of the cavity increase. 
Besides investigating the flow over a rectangular cavity, other cavity shapes also attract researchers to further investigating the effect of cavity geometrical arrangement to the flow structure. Ozalp et al., [18] carried out experiments to investigate the flow structure over the rectangular, triangular and semi-circular cavity with $A R=2$. Idris et al., [19] conducted a numerical study for a shallow semi-ellipse cavity with $A R=4,3$ and 8/3.

Shinha et al., [20] conducted an empirical research of flow inside the rectangular cavities with $A R$ ranging from 0.035 to 2.5 at three $R e$, which are 662, 1342 and 2648 . Since their work provides a good result, it becomes a reference to validate numerical analysis for a quite number of times. Furthermore, Koseff and Street [21] studied the lid-driven cavity for $A R=1$ using laser-Doppleranemometer to measure the flow velocity and a rheoscopic liquid illuminated by laser-light sheets for visualization purpose. They then extended their work for span-width ratio, $W / L=1,2$ and 3 using the same technique [22] at $R e=1000$ to 10,000 .

One of the main components in analyzing driven cavity flow in three dimensional is the existence of Taylor-Görtler-like (TGL) vortices which are identified by Koseff and Street [23] and coined the term in 1984 [21]. It is the longitudinal vortices whose axes lie along the primary flow direction [24] and it becomes significant as the increase of $R e$, depending on the $A R$. Rhee et al., [25] focused on visualizing the TGL longitudinal vortices. According to Eaton [26], TGL may affect heat transfer within the flow. At the same year, Koseff et al., [27] produced evidence that there is a correlation between the passage of the vortices and local heat transfer at the bottom of the cavity. Moreover, Aidun et al., [28] studied the stages that TGL will appear for $W / L=3$. The flow is steady until $R e=825$, then the TGL appear. Besides, the unsteady, longitudinal nature of these formations at $R e=4000$ verified the early study by Iwatsu et al., [29]. They were agreed with Koseff and Street [21]. As the Re increase, the number of pairs of TGL vortices increases for $W / L=3$.

\subsection{Flow Characteristics Effects}

Despite the rising of the interest on the transition Reynolds number, $R e$, it cannot be denied the low to medium $R e$ cavity problem still be analyzed with various additional problem modification and different numerical schemes being applied. Stokes flow is often used to study the fundamental of fluid flow. Stokes flow is a type of fluid flow where the inertial forces are too small compared to the viscous forces where $R e \leq 1$. Kim and Moin [21] and Schreiber and Keller [30] used stokes flow as one of their numerical tests for a square lid-driven cavity. Both provide the same result qualitatively, which the flow is almost symmetrical concerning the centerline, with a single primary vortex and two corners small vortices are visible.

For deep cavity, ranging from 1 to $1 / 3$ of $A R$, increasing of $R e$ made the strength of vortex increase but the size of the first and second vortices decreases [9]. The size differences between both pair of smaller vortices at the bottom corner are noticeable as $R e$ increases. The rotation of the large vortex is influencing which side of the smaller vortices will change in size. Also, Patil et al., [10] conducted a numerical simulation of a two-dimensional rectangular deep cavity with $A R$ ranging from $2 / 3$ to $1 / 4$. The second primary vortex is formed by a rapid transition of an unsteady wall vortex and consistent with previous experiment [8] and numerical study [24, 31] under Stokian flow and high $R e$. The findings also conclude that the value of $R e$ affected the structure of the primary vortex below the top lid drastically, but not much affected by the cavity depth, $H$ as shown in Figure 3 .

Kumar and Agrawal [32] studied the effect of different $A R$ in the driven cavity using the Lattice Boltzmann method with $A R=0.25$ and $R e$ from 100 and reaches over 1000. In the simulations, Kumar and Agrawal [32] predicted four vortices were formed inside the cavity. The finding was the similar result by Lin et al., [33]. The strength of the vortices for $H<2$ is weak, translated by low velocity in 
the horizontal direction. With increasing of $R e$, the strength of the vortices increases, thus, two small vortices at the cavity's bottom wall attached and formed a primary vortex. This event occurred at $R e$ $=400$. Similar findings also can be observed in Patil et al., [10]. This result, however, contradicts with Pandit [34] which he predicted only three vortices formed at $R e=400$.

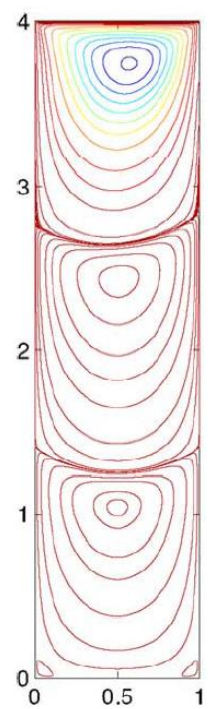

(a)

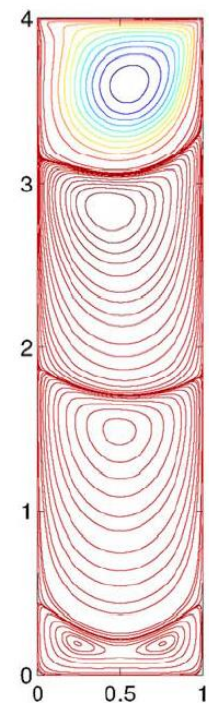

(b)

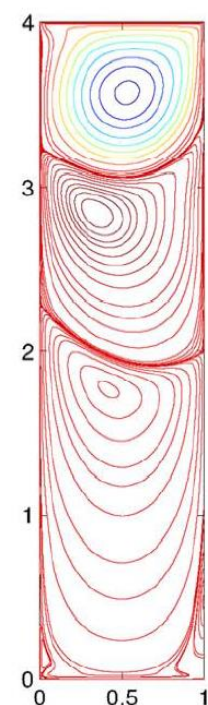

(c)

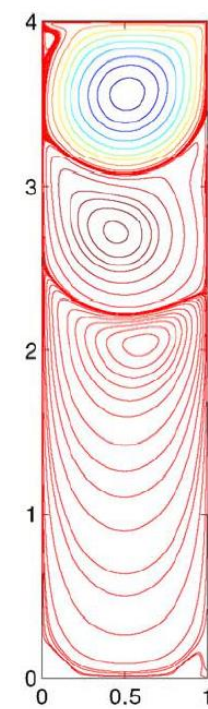

(d)

Fig. 3. Vortex formation for deep cavity flow for $A R=4$ with $R e=(a) 50$ (b) 400, (c) 1000 and (d) 3200 [10]

In a channel with shallow cavity, increasing of Re make the centre of primary vortex drifts off horizontally to the right and flow pattern are no longer symmetrical as in creeping flow due to inertia force effect. In addition, the strength of primary vortex and the size of secondary vortices of the bottom corner of the cavity is also increased. Flow development over a cavity for $A R=4$ have been illustrated by Fang et al., [35] for $R e=50,100$ and 400. The findings show the formation of primary vortex is also highly dependent on $R e$. The vortex is formed from the counter-rotating small vortices at both corners of the cavity's bottom wall. As time goes by, the strength from inertial forces from the incoming flow made the small vortex at the preferred side increase in size. As the $R e$ increase, the impingement point of the vortex to the opposite side of the cavity decrease and finally filling the cavity with central vortex drifted to the right.

In addition, Zdanski et al., [17] investigated the influence of $R e$ in a very shallow cavity with $A R=$ 12. Four sets of $R e$ were used, 147, 294, 442 and 662. For the lowest $R e$, two separates vortices were formed with the backwards-facing step vortex larger than the forward-facing step. As $R e$ increases, the vortices stretched and attached, creating a single larger vortex. They concluded that the position of vortices centre as a function of $R e$.

Studying the transition process from laminar to turbulent flow also attract so many researchers in recent years. Many take the advantages of the simplicity of lid-driven problem as a basis to verify the numerical schemes, physical occurrences, and flow characteristic. Bruneau and Saad [36] used a fourth-order discretization of convection term to solve the Navier-Stokes equations for a variety of Re from 1000 to 10,000. In addition, Peng et al., [37] used Reynolds number starting from 7000 until 11,000 to study the transition. Both used the mark of Hopf bifurcation as the point of transition started. The mark of the first bifurcation is always known as the critical Reynolds number, $R e_{c}$, which the value of $R e$ when the start of the transition of flow from laminar to turbulent. The $R e_{c}$ value is varies depending on the geometry of the boundary. According to Peng et al., [37] the first bifurcation 
occurs at $R e$ equal to 7402 . This result almost the same as the early result of $R e_{c}$ equal to 7500 by Bruneau and Jouron [38]. In comparison, according to Bruneau and Saad [36], the first bifurcation occurs at $R e \approx 8000$, in the same way as Fortin et al., [39] and Auteri et al., [40]. From all these findings, even though different vortices and streamline formed as different $R e$ are applied, it can be concluded that mass transfer of the fluid inside the cavity is increase as $R e$ increases.

\section{Effects of Mixed Convection in Cavity Flow}

Convective heat transfer between the cavity and the forced flow of fluid made the complicated interaction of natural convection because of buoyancy forces within the fluid and the external flow. The convective heat transfer problem in an enclosure has been numerically investigated comprehensively due to its broad application. It can be divided into two main categories, which are open-ended or closed enclosure. The open-ended enclosure is an enclosure where there is a part of the boundary is open to the ambient and closed-ended enclosure is an enclosure where the entire boundary is closed from the ambient. A wide range of related parameters are considered in many studies such as Reynolds number, $R e$, Grashof number, $G r$, Prandtl number, $P r$, and aspect ratio, $A R$ of the enclosure. A review done by Oztop and Dagtekin [41] showed that there are two types of studies related to the flow of fluid and heat transfer in an enclosure. Firstly, it is concerned with horizontal top or bottom with shear wall and secondly, deals with differentially heated side-driven cavities. This categorization also agreed by Cheng [42]. However, majority researchers did not categorize the heat transfer problem in an enclosure in that way as it did not have any concrete reason and only a matter of configuration.

Iwatsu et al., [43] performed extensive studies on the flow and heat transfer of the viscous fluid in the enclosure with aspect ratio $A R=1$. They modelled the cavity as the top wall sliding with a constant velocity while heated. This study is taken conceptually from what have done by Mohamad and Viscanta [44]. They however considered a shallow cavity with the heated bottom wall. The relationship between $R i$ and the flow characteristics has been studied. As $R i \leq 1$, the forced convection becomes dominant, and there are no significant changes in the flow characteristic as the non-heated cavity flow. Contrarily, as $R i>1$, the buoyancy effect becomes dominant. As a result, the effect of the shear at the top only influencing small distances to the cavity center. The middle and the bottom part of the flow of the cavity are stagnant, and the isotherms are almost horizontal. There is almost uniformed temperature distribution can be seen while the only small region on the top where the fluid and heat are well mixed.

Burgos et al., [45] compared the experimental results with the Lattice Boltzmann method to investigate the mixed-convection steady and unsteady laminar flow in a channel with an open square cavity and heated bottom wall from $R e=50$ to 1000 and $R i=0.01$ to 10 . Effect of buoyancy force is negligible for $R i \leq 0.1$ for all values of $R e$ considered. For $R i \geq 1$, the buoyancy effect is important. Combining with higher $R e$ (200), upstream secondary vortex and stratification of the flow into two main recirculating flows. For $R e=500$ and $R i=10$, the flow becomes unsteady, and an oscillatory instability develops. The findings by Abdelmassih et al., [46] showed similar results on when the unsteady occurred on the flow.

\section{Fluids-Particle Interaction}

\subsection{Forces on Particles}

Drag force defines as the forces that acting opposite to the relative motion of any object moving concerning a surrounding fluid. In general, steady-state drag is considered in the analysis of 
particulate flow. The steady-state drag is the drag force that acts on the particle in a velocity field when there is no acceleration occurred to the particle due to relative velocity between the particle and the transmitting fluid. The steady state drag force, $F_{d}$ can be written as

$F_{d}=0.5 \rho_{f} C_{D} A_{P}\left|u_{i}-v_{i}\right|\left(u_{i}-v_{i}\right)$

where $\rho_{f}$ is the density of the fluid, $C_{D}$ is the drag coefficient, $A_{P}$ is the area of the particle projected on a plane normal to the flow direction and $u_{i}$ and $v_{i}$ are the velocities of the continuous fluid phase and particle phase. The value of the $C_{D}$ is primarily a function of Reynolds number, $R e$ defined as

$R e_{p}=\left(\rho_{f} D_{p}\left|u_{i}-v_{i}\right|\right) / \mu$

where $D_{p}$ is the particle diameter and $\mu$ is the dynamic viscosity of the fluid. Since the value of $R e$ here is the value of relative velocity between the particle and the fluid, $R e_{p}$ the notation is used to distinguish between the flow's Reynolds number, $R e$.

The drag coefficient will generally rely on the particle shape and orientation for the flow as well as on the Reynolds number, $R e$ of the flow [47]. There are three regions: Stoke's Law region $\left(R e_{p}<\right.$ $1)$, the transition region, and Newton's law region $\left(R e_{p}>1000\right)$. Well-documented correlations can be used to determine the drag coefficient for each region [47-48]. For non-rotating sphere particles, the drag coefficient variant, $C_{D}$ with $R e$ can be observed in Figure 4.



Fig. 4. Variation of the drag coefficient of a sphere with $\operatorname{Re}$ [47]

\subsection{Effect of Geometrical and Flow Parameters on Particles Dispersion}

The geometrical arrangement of the boundary is an important parameter affecting the dispersion of the particles. The effect of the geometrical arrangement of the boundary to the particle dispersion can be observed in the literature by Saadun et al., [49] and Sidik and Salehi [50] which they used a different shape of the cavity in a channel. Different geometrical boundary changes the development of flow structure inside the cavity, thus improving the dispersion of the particle from the cavity. According to Sidik and Salehi [50], the number of particles dispersed from the triangular cavity despite the range of $R e$ studied is the highest compared to other shapes.

Another factor that influences particle behaviour in the flow is the particle's density itself. Jafari et al., [51] studied the effect of particle density on the dispersion and deposition of the particle in a channel with obstruction for $R e=200$. They have presented that the deposition of particles on the front of blockage decrease as the density decreases. In addition, particles dispersion in the back 
vortices is directly influenced by the density of the particles. The particle distributions showed the preferential pattern of concentration for particles with Stokes number 0.4 and 1 . The centrifugal forces from the vortices push the particles from the higher vorticity region to the lower vorticity region. For higher particles density, however, are barely affected by the vertical flow because of higher inertia.

Similar research was previously done by Brandon and Aggraval [52] but using different variables for the particles. They studied for particles with Stokes number 0.01, 0.1, 0.3, 0.5, 1.0 and 5.0 with $R e=200$ and 2000. It was found out that the increase of Stokes, however, significantly increases the deposition efficiency. Furthermore, the trajectories of the particles at the back of the blockage are dependent on the Stokes number of the particles for constant $R e$. Later, Salmanzadeh et al., [53] studied particle transportation and deposition in a rectangular blockage duct flow with different Stokes number (0.001 to 10$)$. They, however, use the different aspect ratio of the blockage $(0.5,1$ and 2 ) and different blockage ratio (from 0.1 to 0.5 ). They found that different blockage aspect ratio is almost similar, but with increasing of obstruction ratio, the particle deposition on the blockage also increases at all Stokes number.

\subsection{Particle Dispersion and Contaminant Removal from Cavity}

Mickaily and Middleman [54] measured the effectiveness of hydrodynamic cleaning of oil from inside of the pipelines experimentally. Numerical modelling of the pipe surface has included a surface with a cavity have been done previously to demonstrate the surface roughness of the pipe [55]. From the results, it shows that the surface roughness limits the rate of cleaning process due to the recirculation and retention in the surface cavity. In related to the cavity's geometry to the hydrodynamic cleaning, Saadun et al., [49] and Sidik and Salehi [50] investigated the effect of different cavity's shape to the contaminant removal efficiency. Besides using fluid as a foulant, they used particles, trapped inside three different shapes of the cavity; rectangular, semi-circular and triangular. Different shape of cavity gives different cleaning efficiency where the triangular shape has the highest cleaning rate compared to the other shape regardless of the other parameters such as $R e$ of the incoming flow.

Fang [56-57] studied the effect of mix convection to the removal of the contaminant fluid inside the cavity with different $A R$. Despite fluid as the contaminant, Jahanshaloo et al., [58] used particles with the density of the particles are the same as the fluid as a contaminant. Besides the differences, the results show that the convection occurred increases the removal rate of the contaminant from the cavity due to changes in flow structures inside the cavity.

\section{Numerical Analysis}

\subsection{Cubic Interpolation Pseudo-Particle (CIP) Method}

Cubic Interpolated Pseudo-particle (CIP) has been introduced by Takewaki et al., [59] to solve hyperbolic equations. CIP method, like the other numerical scheme, is used to retrieve the information lost within the digitized grid points with the solution closes possible with the actual solution. The simplest way of explaining this by using the example of one-dimensional advection equation (Eq. 3) as described in Yabe et al., [60].

$\frac{\partial u}{\partial t}+c \frac{\partial u}{\partial x}=0$

Eq. (3) then integrate over the interval $\left(x_{i-1 / 2}, x_{i+1 / 2}\right),\left(t_{n}, t_{n+1}\right)$ yielding, 
$\int_{x_{i-1 / 2}}^{x_{i+1 / 2}}\left(u^{n+1}(x)-u^{n}(x)\right) d x=-c \int_{t_{n}}^{t_{n+1}}\left(u_{i+1 / 2}(t)-u_{i-1 / 2}(t)\right) d t$

Where $i$ and $n$ is in the meaning of $t=t_{n}$ and $x=x_{i}$. By interpolating using cubic polynomial to approximate the functional form of $u$ with the range of $\left(x_{i-1 / 2}, x_{i}\right)$, therefore,

$u(x)=a_{i}\left(x_{i}-x_{i-1}\right)^{3}+b_{i}\left(x_{i}-x_{i-1}\right)^{2}+u_{i-1}^{\prime}\left(x_{i}-x_{i-1}\right)+u_{i-1}$

Where $u_{i-1}^{\prime}$ and $u_{i-1}$ are the value and the derivative in the space of $u$ at $x=x_{i-1}$, considering $\Delta x=$ $x_{i}-x_{i-1}$, the coefficient of $a_{i}$ and $b_{i}$ can be defined in term of $u$ and $u^{\prime}$ as

$a_{i}=\frac{\left(u_{i}^{\prime}-u_{i-1}^{\prime}\right) \Delta x-2\left(u_{i}-u_{i-1}\right)}{(\Delta x)^{3}}$

$b_{i}=\frac{-\left(u_{i}^{\prime}+2 u_{i-1}^{\prime}\right) \Delta x+3\left(u_{i}-u_{i-1}\right)}{(\Delta x)^{2}}$

Thus, the final equations are

$u_{i}^{n+1}=a_{i} \xi^{3}+b_{i} \xi^{2}+u_{i}^{\prime} \xi+u_{i}$

$u_{i}^{\prime n+1}=3 a_{i} \xi^{2}+2 b_{i} \xi+u_{i}^{\prime}$

Where $\xi=-c \Delta t$.

When constant velocity is used in the equation, it will become the simple evolution of motion of simple wave with velocity $u$.

Furthermore, since the application of CIP has become more general, it should not be limited to the explicit scheme only. It has been acknowledged that the implicit scheme gives the final desired outcome faster due to its ability to converge more quickly. However, the calculation in the implicit scheme is unstable [61]. The applications of the explicit scheme provide severe inaccurate result. Therefore, there are so many applications that used the implicit scheme in general. Notable cases that can be seen are the simulation of atomization of fuel in the combustion chamber [62], and shallow water problem [63].

\subsection{Fluid-Particle Interaction}

Due to the advantages provided by the Lagrangian approach, it has become a method of choice for many researchers to study a wide range of particle dispersion problem. Chiesa et al., [64] compared the particle distribution in fluidized bed numerically with the experiment. They concluded that the Lagrangian approach can predict the particle distribution inside the bed. In addition, Kosinski et al., [65] studied the particle dispersion inside the enclosure using Lagrangian approach. Comparison of their validation study with experiment work by Tsorng et al., [66] showed that the Lagrangian approach can provide high accuracy numerical results. On the other hand, Saadun et al., [49] and Jahanshaloo et al., [58] studied the particle dispersion from the cavity. Recently, Sahak et al., [67] investigated the behaviour of the solid particles in the dispersion process from the cavity inside the channel at the various combination of geometrical, flow and particles parameters. They 
findings show that the particles are dependent on the flow characteristics inside the cavity. The particles move as a result of the drag force of the particles from the fluid.

\subsection{Particle-Particle Interaction}

Cundall and Shack [68] is the first to develop the soft-particle approach in the simulation of granular dynamics. In this approach, the particle is deformed when interacts with other bodies, another particle, wall or the surrounding fluid. These deformations that occur are used to measure other parameters between particles, such as frictional, elastic and plastic forces. This makes the softparticles approaches capable of handling multiple particles contacts, thus made it suitable for modelling the quasi-static systems. The feature makes the soft-particles methods being extensively studied to understanding the phenomena such as mixing and granulation process, particle packing and heaping or pilling process.

In contrast, hard-particle approaches explained that when the collision occurred, it is instantaneous with a single collision occur at a time. Due to its short moment of collisions, the forces among particles are often neglected. Therefore, it is useful in the rapid granular flow analysis such as hydraulic or pneumatic transport of dust, fluidized bed and slurry transport of powders.

Soft-sphere particle approaches, in general, explained that the contact point between two colliding particles is not a single but rather a finite region due to deformation of the particles. Softsphere particle approach, it can be the same as the two slightly overlapped rigid bodies, as shown in Figure 5. The distribution of the contact traction in this region can be divided into a component in the contact plane and normal to the plane so that the two components of the contact force which are normal and tangential forces can be established. Thus, it will lead to the total torque and forces that have occurred on the particle. Since it is so difficult in determining the distribution of contact traction in this region, Cundall and Strack [68] proposed the most simple and intuitive models that called spring-dashpot model where the spring is modelled after an elastic deformation and for viscous dissipation, the damper was used. This linear spring model also was being used by other researchers like Di Renzo and Di Maio [69] but without the inclusion of damper. Other commonly used models for the soft-sphere collision between spherical particles are Walton and Braun's model [70] and simplified Hertz-Mindin and Dereisewicz model [71].

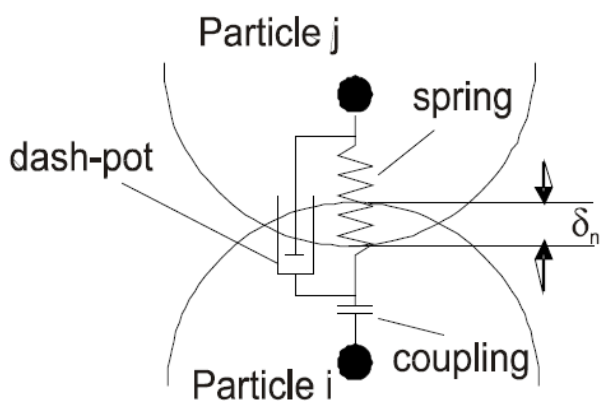

a) Normal force

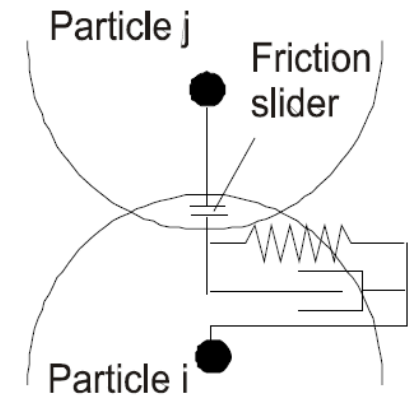

b) Tangential force

Fig. 5. Soft-sphere model: (a) normal force and (b) tangential force [47]

The hard-sphere particles approaches are quite simple to use but only applicable to binary collisions. The interaction forces are considered to be impulsive and therefore, the momentum exchange between particles only happens when the collision occurs. The relationship between the particle velocity before and after the collision is clearly stated by using the coefficient of restitution and the coefficient of friction [47]. In addition, it is adequate to only consider binary collision and not 
the collisions of multiples particles if the particles are well distributed in the domain. According to the Hoomans et al., [72], to model the hard-sphere collision model, several crucial factors need to be taken into considerations which are the particle shape, the deformation history during the collision and the collision disposition of the particle.

However, Hoomans et al., [72] believed that the assumption that they have made could only be real for the relatively coarse particle $(100 \mu \mathrm{m})$. Beforehand, other researchers like Buyevich [73] and Nieuwland [74] assumed the nature of the collision that similar to Hoomans et al., [72] in their works.

\section{Conclusions}

This paper presents a review studies on the particle dispersion from the cavity flow. The outcomes from this literature are summarized as follow.

I. The cavity flow regime and mass transfer vary with the geometry of the cavity (aspect ratio, $A R$ ), Reynolds number, $R e$ of the incoming flow and the heat transfer from the cavity.

II. Particles dispersion and deposition are related to the $R e$ of the flow. The concentration of the dispersion is higher at the higher vorticity region. However, given the density of the particles, the greater density of the particles is hardly influenced by the flow due to inertial forces.

III. The presence of heat transfers in the flow also affecting the dispersion of the particles.

IV. The particles collision can be solved by two main approaches, hard-sphere particle and soft-sphere particles approach. The hard-sphere particles approaches are quite simple to use but only applicable to binary collisions.

\section{Acknowledgement}

This research was not funded by any grant.

\section{References}

[1] Yu, Haijun, and Pingwen Zhang. "A kinetic-hydrodynamic simulation of microstructure of liquid crystal polymers in plane shear flow." Journal of non-newtonian fluid mechanics 141, no. 2-3 (2007): 116-127. https://doi.org/10.1016/j.jnnfm.2006.09.005

[2] Oke, Timothy R. Boundary layer climates. Routledge, 2002. https://doi.org/10.4324/9780203407219

[3] Akubue, Jideofor Anselm. "Effects of Street Geometry on Airflow Regimes for Natural Ventilation in Three Different Street Configurations in Enugu City." In Different Strategies of Housing Design. IntechOpen, 2019.

[4] Charwat, A. F., J. N. Roos, F. C. Dewey Jr, and J. A. Hitz. "An investigation of separated flows-Part I: The pressure field." Journal of the Aerospace Sciences 28, no. 6 (1961): 457-470. https://doi.org/10.2514/8.9037

[5] Pan, Frank, and Andreas Acrivos. "Steady flows in rectangular cavities." Journal of Fluid Mechanics 28, no. 4 (1967): 643-655.

https://doi.org/10.1017/S002211206700237X

[6] Shankar, P. N., and M. D. Deshpande. "Fluid mechanics in the driven cavity." Annual review of fluid mechanics 32 , no. 1 (2000): 93-136. https://doi.org/10.1146/annurev.fluid.32.1.93

[7] Joseph, D. D., D. A. Nield, and G. Papanicolaou. "Nonlinear equation governing flow in a saturated porous medium." Water Resources Research 18, no. 4 (1982): 1049-1052. https://doi.org/10.1029/WR018i004p01049

[8] Hellou, Mustapha, and Madeleine Coutanceau. "Cellular Stokes flow induced by rotation of a cylinder in a closed channel." Journal of Fluid Mechanics 236 (1992): 557-577. https://doi.org/10.1017/S0022112092001538 
[9] Cheng, M., and K. C. Hung. "Vortex structure of steady flow in a rectangular cavity." Computers \& fluids 35, no. 10 (2006): 1046-1062. https://doi.org/10.1016/i.compfluid.2005.08.006

[10] Patil, D. V., K. N. Lakshmisha, and B. Rogg. "Lattice Boltzmann simulation of lid-driven flow in deep cavities." Computers \& fluids 35, no. 10 (2006): 1116-1125. https://doi.org/10.1016/i.compfluid.2005.06.006

[11] Shankar, P. N. "The eddy structure in Stokes flow in a cavity." Journal of Fluid mechanics 250 (1993): 371-383. https://doi.org/10.1017/S0022112093001491

[12] LAVAN, Z., and UB MEHTA. "Flow in a two-dimensional channel with a rectangular cavity(Flow structure in rectangular cavity in lower wall of two dimensional channel for various aspect ratios and Reynolds numbers)." ASME, TRANSACTIONS, SERIES E-JOURNAL OF APPLIED MECHANICS 36 (1969): 897-901. https://doi.org/10.1115/1.3564799

[13] Chang, Ho Nam, Hwa Won Ryu, Deug Ho Park, Yong Seok Park, and Joong Kon Park. "Effect of external laminar channel flow on mass transfer in a cavity." International journal of heat and mass transfer 30, no. 10 (1987): 2137 2149.

https://doi.org/10.1016/0017-9310(87)90092-5

[14] Taneda, Sadatoshi. "Visualization of separating Stokes flows." Journal of the Physical Society of Japan 46, no. 6 (1979): 1935-1942. https://doi.org/10.1143/JPSJ.46.1935

[15] Shen, C., and J. M. Floryan. "Low Reynolds number flow over cavities." The Physics of fluids 28, no. 11 (1985): $3191-$ 3202. https://doi.org/10.1063/1.865366

[16] Moffatt, H. Keith. "Viscous and resistive eddies near a sharp corner." Journal of Fluid Mechanics 18, no. 1 (1964): 1-18. https://doi.org/10.1017/S0022112064000015

[17] Zdanski, P. S. B., M. A. Ortega, and Nide GCR Fico Jr. "Numerical study of the flow over shallow cavities." Computers \& fluids 32, no. 7 (2003): 953-974. https://doi.org/10.1016/S0045-7930(02)00067-1

[18] Ozalp, Coskun, A. Pinarbasi, and B. Sahin. "Experimental measurement of flow past cavities of different shapes." Experimental Thermal and Fluid Science 34, no. 5 (2010): 505-515. https://doi.org/10.1016/j.expthermflusci.2009.11.003

[19] Idris, M. S., M. A. M. Irwan, and N. M. M. Ammar. "Steady state vortex structure of lid driven flow inside shallow semi ellipse cavity." Journal of Mechanical Engineering and Sciences 2 (2012): 206-216. https://doi.org/10.15282/jmes.2.2012.8.0019

[20] Sinha, S. N., A. K. Gupta, and M. M. Oberai. "Laminar separating flow over backsteps and cavities. II-Cavities." AIAA Journal 20, no. 3 (1982): 370-375. https://doi.org/10.2514/3.7918

[21] Koseff, J. R., and R. L. Street. "The lid-driven cavity flow: a synthesis of qualitative and quantitative observations." (1984): 390-398. https://doi.org/10.1115/1.3243136

[22] Koseff, J. R., and R. L. Street. "Visualization studies of a shear driven three-dimensional recirculating flow." (1984): 21-27. https://doi.org/10.1115/1.3242393

[23] Koseff, J. R., R. L. Street, P. M. Gresho, C. D. Upson, J. A. C. Humphrey, and W. M. To. Three-dimensional lid-driven cavity flow: experiment and simulation. No. CONF-830803-11. Stanford Univ., CA (USA). Dept. of Civil Engineering; Lawrence Livermore National Lab., CA (USA); California Univ., Berkeley (USA). Dept. of Mechanical Engineering, 1983.

[24] Shankar, P. N., and M. D. Deshpande. "Fluid mechanics in the driven cavity." Annual review of fluid mechanics 32, no. 1 (2000): 93-136. https://doi.org/10.1146/annurev.fluid.32.1.93

[25] Rhee, H. S., J. R. Koseff, and R. L. Street. "Flow visualization of a recirculating flow by rheoscopic liquid and liquid crystal techniques." Experiments in Fluids 2, no. 2 (1984): 57-64.

[26] Eaton, J. K. "Heat transfer effects of longitudinal vortices embedded in a turbulent boundary layer." stan (1986).

[27] Koseff, J. R., H. Rhee, and R. L. Street. Mixing in a stratified recirculating flow. No. CONF-8308103-2. Stanford Univ., CA (USA), 1983.

[28] Aidun, Cyrus K., N. G. Triantafillopoulos, and J. D. Benson. "Global stability of a lid-driven cavity with throughflow: Flow visualization studies." Physics of Fluids A: Fluid Dynamics 3, no. 9 (1991): 2081-2091. 


\section{https://doi.org/10.1063/1.857891}

[29] Iwatsu, Reima, Katsuya Ishii, Tetuya Kawamura, Kunio Kuwahara, and Jae Min Hyun. "Numerical simulation of three-dimensional flow structure in a driven cavity." Fluid Dynamics Research 5, no. 3 (1989): 173.

https://doi.org/10.1016/0169-5983(89)90020-8

[30] Schreiber, Rob, and Herbert B. Keller. "Driven cavity flows by efficient numerical techniques." Journal of Computational Physics 49, no. 2 (1983): 310-333. https://doi.org/10.1016/0021-9991(83)90129-8

[31] Gustafson, K., and K. Halasi. "Vortex dynamics of cavity flows." Journal of Computational Physics 64, no. 2 (1986): 279-319. https://doi.org/10.1016/0021-9991(86)90035-5

[32] Kumar, Anil, and S. P. Agrawal. "Mathematical and simulation of lid driven cavity flow at different aspect ratios using single relaxation time lattice boltzmann technique." American Journal of Theoretical and Applied Statistics 2, no. 3 (2013): 87-93. https://doi.org/10.11648/j.ajtas.20130203.17

[33] Lin, Li-Song, Yi-Cheng Chen, and Chao-An Lin. "Multi relaxation time lattice Boltzmann simulations of deep lid driven cavity flows at different aspect ratios." Computers \& Fluids 45, no. 1 (2011): 233-240. https://doi.org/10.1016/i.compfluid.2010.12.012

[34] Pandit, Swapan K. "On the use of compact streamfunction-velocity formulation of steady Navier-Stokes equations on geometries beyond rectangular." Journal of Scientific Computing 36, no. 2 (2008): 219-242. https://doi.org/10.1007/s10915-008-9186-8

[35] Fang, L. C., D. Nicolaou, and J. W. Cleaver. "Transient removal of a contaminated fluid from a cavity." International journal of heat and fluid flow 20, no. 6 (1999): 605-613. https://doi.org/10.1016/S0142-727X(99)00050-8

[36] Bruneau, Charles-Henri, and Mazen Saad. "The 2D lid-driven cavity problem revisited." Computers \& fluids 35, no. 3 (2006): 326-348. https://doi.org/10.1016/i.compfluid.2004.12.004

[37] Peng, Yih-Ferng, Yuo-Hsien Shiau, and Robert R. Hwang. "Transition in a 2-D lid-driven cavity flow." Computers \& Fluids 32, no. 3 (2003): 337-352.

https://doi.org/10.1016/S0045-7930(01)00053-6

[38] Bruneau, Charles-Henri, and Claude Jouron. "An efficient scheme for solving steady incompressible Navier-Stokes equations." Journal of Computational Physics 89, no. 2 (1990): 389-413.

https://doi.org/10.1016/0021-9991(90)90149-U

[39] Fortin, A., M. Jardak, J. J. Gervais, and R. Pierre. "Localization of Hopf bifurcations in fluid flow problems." International Journal for Numerical Methods in Fluids 24, no. 11 (1997): 1185-1210. https://doi.org/10.1002/(SICI)1097-0363(19970615)24:11<1185::AID-FLD535>3.0.CO;2-X

[40] Auteri, F. R. A. N. C. O., N. I. C. O. L. A. Parolini, and L. Quartapelle. "Numerical investigation on the stability of singular driven cavity flow." Journal of Computational Physics 183, no. 1 (2002): 1-25. https://doi.org/10.1006/jcph.2002.7145

[41] Oztop, Hakan F., and Ihsan Dagtekin. "Mixed convection in two-sided lid-driven differentially heated square cavity." International Journal of Heat and mass transfer 47, no. 8-9 (2004): 1761-1769. https://doi.org/10.1016/i.ijheatmasstransfer.2003.10.016

[42] Cheng, T. S. "Characteristics of mixed convection heat transfer in a lid-driven square cavity with various Richardson and Prandtl numbers." International Journal of Thermal Sciences 50, no. 2 (2011): 197-205. https://doi.org/10.1016/j.ijthermalsci.2010.09.012

[43] Iwatsu, Reima, Jae Min Hyun, and Kunio Kuwahara. "Mixed convection in a driven cavity with a stable vertical temperature gradient." International Journal of Heat and Mass Transfer 36, no. 6 (1993): 1601-1608. https://doi.org/10.1016/S0017-9310(05)80069-9

[44] Mohamad, A. A., and R. Viskanta. "Transient low Prandtl number fluid convection in a lid-driven cavity." Numerical Heat Transfer 19, no. 2 (1991): 187-205. https://doi.org/10.1080/10407789108944845

[45] Burgos, J., I. Cuesta, and C. Salueña. "Numerical study of laminar mixed convection in a square open cavity." International Journal of Heat and Mass Transfer 99 (2016): 599-612.

https://doi.org/10.1016/i.ijheatmasstransfer.2016.04.010

[46] Abdelmassih, Gorg, Anton Vernet, and Jordi Pallares. "Steady and unsteady mixed convection flow in a cubical open cavity with the bottom wall heated." International Journal of Heat and Mass Transfer 101 (2016): 682-691. https://doi.org/10.1016/i.ijheatmasstransfer.2016.05.074 
[47] Crowe, Clayton T., John D. Schwarzkopf, Martin Sommerfeld, and Yutaka Tsuji. Multiphase flows with droplets and particles. CRC press, 2011.

https://doi.org/10.1201/b11103

[48] Cheng, Nian-Sheng. "Comparison of formulas for drag coefficient and settling velocity of spherical particles." Powder Technology 189, no. 3 (2009): 395-398.

https://doi.org/10.1016/i.powtec.2008.07.006

[49] Saadun, Mohd Noor Asril, C. S. Azwadi, M. N. Hazwani, Mohamad Shukri Zakaria, Muhammad Zaidan Abdul Manaf, Mohd Hanafi, and Mohd Hafidzal. "Numerical analysis on the effects of cavity geometry with heat towards contaminant removal." In Applied Mechanics and Materials, vol. 393, pp. 851-856. Trans Tech Publications Ltd, 2013.

https://doi.org/10.4028/www.scientific.net/AMM.393.851

[50] Sidik, Nor Azwadi Che, and Mehran Salehi. "Eulerian-Lagrangian numerical scheme for contaminant removal from different cavity shapes." Arabian Journal for Science and Engineering 39, no. 4 (2014): 3181-3189. https://doi.org/10.1007/s13369-013-0886-5

[51] Jafari, Saeed, Mazyar Salmanzadeh, Mohammad Rahnama, and Goodarz Ahmadi. "Investigation of particle dispersion and deposition in a channel with a square cylinder obstruction using the lattice Boltzmann method." Journal of Aerosol Science 41, no. 2 (2010): 198-206.

https://doi.org/10.1016/i.jaerosci.2009.10.005

[52] Brandon, Daniel J., and S. K. Aggarwal. "A numerical investigation of particle deposition on a square cylinder placed in a channel flow." Aerosol Science \& Technology 34, no. 4 (2001): 340-352. https://doi.org/10.1080/02786820121279

[53] Salmanzadeh, M., M. Rahnama, and G. Ahmadi. "Particle transport and deposition in a duct flow with a rectangular obstruction." Particulate Science and Technology 25, no. 5 (2007): 401-412.

https://doi.org/10.1080/02726350701487181

[54] Mickaily, Elizabeth S., and Stanley Middleman. "Hydrodynamic cleaning of a viscous film from the inside of a long tube." AlChE journal 39, no. 5 (1993): 885-893.

https://doi.org/10.1002/aic.690390517

[55] MICKAILY, ELIZABETH S., STANLEY MIDDLEMAN, and MERRY ALLEN. "Viscous flow over periodic surfaces." Chemical Engineering Communications 117, no. 1 (1992): 401-414.

https://doi.org/10.1080/00986449208936078

[56] Fang, Lih-Chuan. "Effect of mixed convection on transient hydrodynamic removal of a contaminant from a cavity." International journal of heat and mass transfer 46, no. 11 (2003): 2039-2049. https://doi.org/10.1016/S0017-9310(02)00507-0

[57] Fang, Lih-chuan. "Effect of duct velocity profile and buoyancy-induced flow on efficiency of transient hydrodynamic removal of a contaminant from a cavity." International journal for numerical methods in fluids 44, no. 12 (2004): 1389-1404.

https://doi.org/10.1002/fld.676

[58] Jahanshaloo, Leila, Nor Azwadi Che Sidik, Shahin Salimi, and Arman Safdari. "The use of thermal lattice Boltzmann numerical scheme for particle-laden channel flow with a cavity." Numerical Heat Transfer, Part A: Applications 66, no. 4 (2014): 433-448. https://doi.org/10.1080/10407782.2014.884898

[59] Takewaki, H., A. Nishigushi, and T. Yabe. "Cubic interpolated pseudo-particle method (CIP) for solving hyperbolic type equation." National Institute for Fusion Science. NII-Electronic Library Services (1985). https://doi.org/10.1016/0021-9991(85)90085-3

[60] Yabe, T., K. Takizawa, M. Chino, M. Imai, and C. C. Chu. "Challenge of CIP as a universal solver for solid, liquid and gas." International journal for numerical methods in fluids 47, no. 6-7 (2005): 655-676. https://doi.org/10.1002/fld.830

[61] Ida, Masato, and Takashi Yabe. "Implicit CIP (Cubic-Interpolated Propagation) method in one dimension." Computer physics communications 92, no. 1 (1995): 21-26. https://doi.org/10.1016/0010-4655(95)92245-C

[62] Desjardins, Olivier, Vincent Moureau, and Heinz Pitsch. "An accurate conservative level set/ghost fluid method for simulating turbulent atomization." Journal of computational physics 227, no. 18 (2008): 8395-8416. https://doi.org/10.1016/i.jcp.2008.05.027

[63] Bermúdez, Alfredo, Alain Dervieux, Jean-Antoine Desideri, and M. Elena Vázquez. "Upwind schemes for the twodimensional shallow water equations with variable depth using unstructured meshes." Computer methods in applied mechanics and engineering 155, no. 1-2 (1998): 49-72.

https://doi.org/10.1016/S0045-7825(97)85625-3 
[64] Chiesa, Matteo, Vidar Mathiesen, Jens A. Melheim, and Britt Halvorsen. "Numerical simulation of particulate flow by the Eulerian-Lagrangian and the Eulerian-Eulerian approach with application to a fluidized bed." Computers \& chemical engineering 29, no. 2 (2005): 291-304.

https://doi.org/10.1016/i.compchemeng.2004.09.002

[65] Kosinski, Pawel, Anna Kosinska, and Alex Christian Hoffmann. "Simulation of solid particles behaviour in a driven cavity flow." Powder Technology 191, no. 3 (2009): 327-339. https://doi.org/10.1016/i.powtec.2008.10.025

[66] Tsorng, S. J., H. Capart, J. S. Lai, and D. L. Young. "Three-dimensional tracking of the long time trajectories of suspended particles in a lid-driven cavity flow." Experiments in Fluids 40, no. 2 (2006): 314-328. https://doi.org/10.1007/s00348-005-0070-0

[67] Sahak, A. Ahmad Sofianuddin, Nor Azwadi Che Sidik, and Siti Nurul Akmal Yusof. "Cubic Interpolation PseudoParticle Navier-Stokes Formulation Method for Solid Particle-Fluid Interaction in Channel Flow with Cavity." Journal of Advanced Research in Materials Science 70, no. 1 (2020): 1-17. https://doi.org/10.37934/arms.70.1.117

[68] Cundall, Peter A., and Otto DL Strack. "A discrete numerical model for granular assemblies." geotechnique 29, no. 1 (1979): 47-65. https://doi.org/10.1680/geot.1979.29.1.47

[69] Di Renzo, Alberto, and Francesco Paolo Di Maio. "Comparison of contact-force models for the simulation of collisions in DEM-based granular flow codes." Chemical engineering science 59, no. 3 (2004): 525-541. https://doi.org/10.1016/i.ces.2003.09.037

[70] Walton, Otis R. "Numerical simulation of inclined chute flows of monodisperse, inelastic, frictional spheres." Mechanics of materials 16, no. 1-2 (1993): 239-247. https://doi.org/10.1016/0167-6636(93)90048-V

[71] Langston, P. A., U. Tüzün, and D. M. Heyes. "Continuous potential discrete particle simulations of stress and velocity fields in hoppers: transition from fluid to granular flow." Chemical Engineering Science 49, no. 8 (1994): 1259-1275. https://doi.org/10.1016/0009-2509(94)85095-X

[72] Hoomans, B. P. B., J. A. M. Kuipers, Willem J. Briels, and Willibrordus Petrus Maria van Swaaij. "Discrete particle simulation of bubble and slug formation in a two-dimensional gas-fluidised bed: a hard-sphere approach." Chemical Engineering Science 51, no. 1 (1996): 99-118. https://doi.org/10.1016/0009-2509(95)00271-5

[73] Buyevich, Yu A., and S. K. Kapbasov. "Fluid dynamics of coarse dispersions." In Advances in Engineering Fluid Mechanics: Multiphase Reactor and Polymerization System Hydrodynamics, pp. 119-166. Gulf Professional Publishing, 1996. https://doi.org/10.1016/B978-088415497-6/50009-7

[74] Nieuwland, Jelle Johan. "Hydrodynamic modelling of gas-solid two-phase flows." (1996): 0516-0516. 